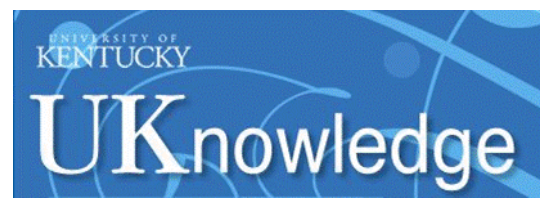

University of Kentucky

UKnowledge

2-1-1985

\title{
The Hydrogen Line Spectra of Narrow-Line Radio Galaxies
}

\author{
Gary J. Ferland \\ University of Kentucky, gary@uky.edu \\ Donald E. Osterbrock \\ University of California - Santa Cruz
}

Follow this and additional works at: https://uknowledge.uky.edu/physastron_facpub

Part of the Astrophysics and Astronomy Commons, and the Physics Commons

Right click to open a feedback form in a new tab to let us know how this document benefits you.

\section{Repository Citation}

Ferland, Gary J. and Osterbrock, Donald E., "The Hydrogen Line Spectra of Narrow-Line Radio Galaxies" (1985). Physics and Astronomy Faculty Publications. 176.

https://uknowledge.uky.edu/physastron_facpub/176

This Article is brought to you for free and open access by the Physics and Astronomy at UKnowledge. It has been accepted for inclusion in Physics and Astronomy Faculty Publications by an authorized administrator of UKnowledge. For more information, please contact UKnowledge@lsv.uky.edu. 


\section{The Hydrogen Line Spectra of Narrow-Line Radio Galaxies}

Digital Object Identifier (DOI)

http://dx.doi.org/10.1086/162867

\section{Notes/Citation Information}

Published in The Astrophysical Journal, v. 289, no. 1, p. 105-108.

(c) 1985. The American Astronomical Society. All rights reserved.

The copyright holder has granted permission for posting the article here. 


\author{
Gary J. FerLand ${ }^{2}$ \\ Department of Physics and Astronomy, University of Kentucky \\ AND \\ DONALD E. OSTERBROCK \\ Lick Observatory, Board of Studies in Astronomy and Astrophysics, \\ University of California, Santa Cruz \\ Received 1984 June 11; accepted 1984 August 17
}

\begin{abstract}
We report the results of the first detection of Ly $\alpha$ in a narrow-line radio galaxy. Nearly simultaneous optical and UV observations of 3C 192 and 3C 223 allow the measurement of both Balmer and Lyman decrements. These line ratios are approximate functions of the interstellar reddening and of a parameter which is proportional to the amount of $\mathrm{H}$ I collisional excitation present. The reddening of 3C 192 is slightly larger than that due to our Galaxy, although 3C 223 may have a larger value. Both galaxies have intrinsic Balmer and Lyman decrements which are significantly steeper than case $\mathrm{B}$, suggesting that the gas is photoionized by a fairly hard X-ray continuum. The deduced values of $\mathrm{L} \alpha / \mathrm{H} \beta$ and $\mathrm{H} \alpha / \mathrm{H} \beta$ compare favorably with predictions of recent models.
\end{abstract}

Subject headings: radiation mechanisms - radio sources: galaxies - ultraviolet: spectra

\section{INTRODUCTION}

Narrow-line radio galaxies (NLRG) are the radio-loud cousins of classical Seyfert 2 galaxies (see, e.g., Cohen and Osterbrock 1981). Both groups are characterized by the presence of strong narrow emission lines, and by the absence of broad components to permitted lines. We have chosen to study the $\mathrm{H}_{\mathrm{I}}$ lines in these objects for several reasons, the first being a practical one; the absence of contamination by broad lines makes it possible to obtain a reliable narrow-line region spectrum. Comparison of measured $\mathrm{H} \alpha, \mathrm{H} \beta$, and $\mathrm{Ly} \alpha$ intensities gives quantitative information on the excitation mechanism and extinction. Understanding of the emission line regions in NLRGs may have application to the low density gas in the more complicated Seyfert 1 and broad-line radio galaxies.

\section{OBSERVATIONS}

Nearly simultaneous observations of the narrow-line radio galaxies 3C 192 and 3C 223 were obtained using the International Ultraviolet Explorer and Lick Observatory Shane telescopes. $I U E$ was used in the low $(\delta \lambda \approx 7 \AA)$ dispersion mode to cover the short wave (1200-2000 $\AA$ ) spectrum; details of the observations are listed in Table 1. Optical spectra of these objects have been discussed elsewhere (Costero and Osterbrock 1977; Cohen and Osterbrock 1981), and we focus attention on the absolute fluxes in the optical region and the $I U E$ spectra here.

Only the Ly $\alpha$ line was positively detected in our ultraviolet spectra (Fig. 1). In making this figure the observed wavelengths were first corrected for redshift (3C 192, $z=0.0598 ; 3 C 223$, $z=0.1367$ ), and the regions near Ly $\alpha$ were then plotted on a common radial velocity scale. The velocity resolution of the IUE spectrometer is $\sim 1500 \mathrm{~km} \mathrm{~s}^{-1}$ at the wavelength of Ly $\alpha$, and the entrance aperture projects to roughly half this. As a

\footnotetext{
${ }^{1}$ Lick Observatory Bulletin, No. 994.

${ }^{2}$ Guest Observer with the International Ultraviolet Explorer Satellite, which is sponsored and operated by NASA, the SERC of the United Kingdom, and the European Space Agency.
}

result the zero point of the wavelength scale is uncertain by $\sim 700 \mathrm{~km} \mathrm{~s}^{-1}$ due to uncertainty in the position of the sources within the entrance aperture. Both emission lines are entirely instrumentally broadened, consistent with the optical line widths. These observations are the first detection of $\mathrm{Ly} \alpha$ in a NLRG. The ultraviolet continua were too weak to be detected in both objects.

The fluxes in $\mathrm{H} \alpha$ and $\mathrm{H} \beta$ were measured with the Shane $3 \mathrm{~m}$ reflector, especially for comparison with the $I U E$ measurements. The radio galaxy 3C 192 was observed on 1984 March $7 / 8$, using the image tube-image dissector scanner and standard reduction techniques (see, e.g., Osterbrock 1981), except

TABLE 1

OBSERVATIONS

\begin{tabular}{|c|c|c|}
\hline Parameter & 3C 192 & $3 \mathrm{C} 223$ \\
\hline \multicolumn{3}{|c|}{ Ultraviolet } \\
\hline $\begin{array}{l}\text { Image } \ldots \ldots \ldots \ldots \ldots \\
\text { Date }(\text { JD } 2,445,000+) \ldots \ldots \ldots \\
\text { Exposure (minutes) } \ldots \ldots \ldots \ldots \\
f(\text { Ly } \alpha) \times 10^{14} \\
\quad\left(\text { ergs cm } \text { cm }^{-2} \mathrm{~s}^{-1}\right) \ldots \ldots \ldots \ldots\end{array}$ & $\begin{array}{l}\text { SWP } 22441 \\
\quad 768.03 \\
256 \\
11( \pm 0.2 \text { dex })\end{array}$ & $\begin{array}{c}\text { SWP } 22438 \\
767.01 \\
365 \\
\\
3.3( \pm 0.3 \mathrm{dex})\end{array}$ \\
\hline \multicolumn{3}{|c|}{ Optical } \\
\hline 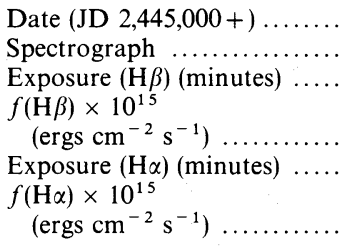 & $\begin{array}{c}767.69 \\
\text { IDS } \\
144 \\
4.2 \pm 0.6 \\
\frac{48}{14.7} \pm 1.5\end{array}$ & $\begin{array}{c}788.71 \\
\text { CCD } \\
60 \\
3.6 \pm 0.6 \\
\cdots\end{array}$ \\
\hline 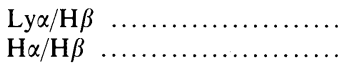 & $\begin{array}{l}26( \pm 0.2 \mathrm{dex}) \\
3.56\end{array}$ & $9.2( \pm 0.3 \mathrm{dex})$ \\
\hline
\end{tabular}

a Balmer decrement from Cohen and Osterbrock 1981. 


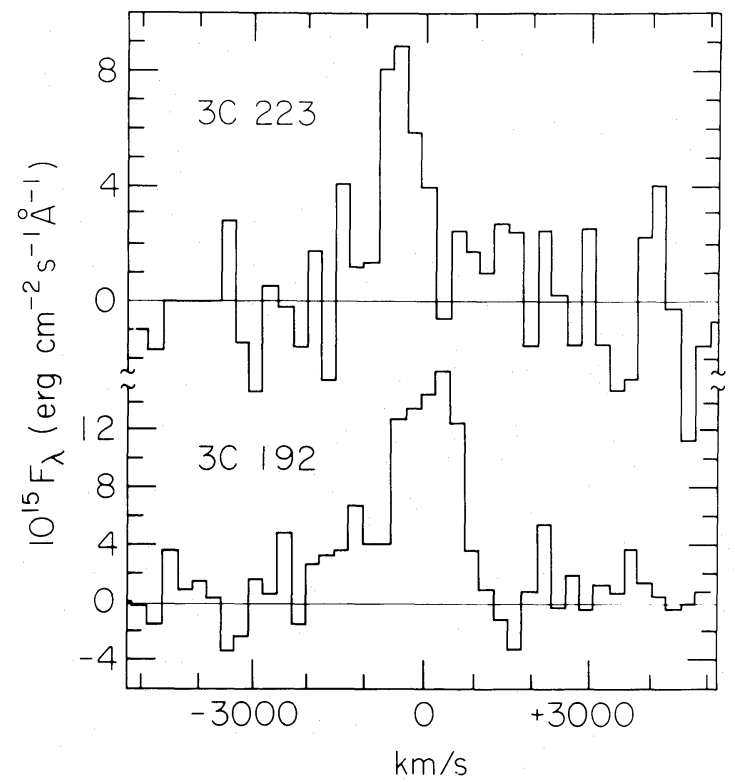

FIG. 1.-IUE spectra of 3C 192 and 3C 223. The region near Ly $\alpha$ is shown in these panels. The lines are instrumentally broadened, and the wavelength scale has a zero point uncertainty of $\sim 700 \mathrm{~km} \mathrm{~s}^{-1}$. No other lines were detected in our IUE data.

that entrance apertures $8^{\prime \prime} \times 8^{\prime \prime}$ were used for both the object and the standard stars, to eliminate possible errors due to differential refraction and to differences in seeing during the night. The normal-dispersion 600 line $\mathrm{mm}^{-1}$ grating was used. A 96 minute exposure was obtained in the blue region, including $\mathrm{H} \beta$, plus a 48 minute exposure of the red region, including both $\mathrm{H} \beta$ and $\mathrm{H} \alpha$. The measured results, expressed as fluxes in the rest system of the Sun, are also given in Table 1 . The major uncertainty in $\mathrm{H} \beta$ results from the fact that it is a faint line; in $\mathrm{H} \alpha$, because it must be deblended from [N II] $\lambda \lambda 6583,6548$. The estimated probable errors given in the table are based on comparison of the two $\mathrm{H} \beta$ measurements, as well as on previous experience with similar absolute-flux measurements. The Balmer line ratio $\mathrm{H} \alpha / \mathrm{H} \beta=3.56$ listed in Table 1 is the straight average of the values 3.50 from the present determination and 3.61 from Costero and Osterbrock (1977).

The radio galaxy 3C 223 was observed on 1984 March $28 / 29$, using the CCD spectrograph (Lauer et al. 1984) with a long slit $8^{\prime \prime}$ wide. Two exposures, each 30 minutes long, were obtained of the region including $\mathrm{H} \beta$ with the 420 grooves $\mathrm{mm}^{-1}$ grating, giving a resolution of approximately $11 \AA$ (FWHM). The resulting measured flux for $\mathrm{H} \beta$ is listed in Table 1 . We have very little experience with absolute spectrophotometry with the CCD and the probable error given is no more than an estimate. Since $\mathrm{H} \alpha$ was not measured, the $\mathrm{H} \alpha / \mathrm{H} \beta$ directly from Cohen and Osterbrock (1981) was used for $3 \mathrm{C} 223$.

\section{HYDROGEN EMISSION FROM PHOTOIONIZED NEBULAE}

Hydrogen emission from a low-density gas, photoionized by a thermal continuum, is well understood (cf., e.g., Osterbrock 1974). Emission lines are formed by pure recombination in such objects as $\mathrm{H}$ II regions and planetary nebulae; collisions from the ground state are unimportant because the temperature is very low in regions where hydrogen has an appreciable neutral fraction, and collisional excitation from excited states is negligible due to the relatively low populations of these states. For all practical cases the optical depth in the Lyman lines is large enough for case B to be a good approximation. Under these conditions the spectrum is sensitive mostly to the electron density. In the low-density case $\left(N_{e} \ll\right.$ $10^{4} \mathrm{~cm}^{-3}$ ), all captures to $2 \mathrm{~s}$ decay via two-photon emission, while at high $\left(N_{e} \gg 10^{4} \mathrm{~cm}^{-3}\right)$ densities $2 s-2 p$ collisions are faster than $2 s-1 s$ decays and most $2 s$ captures eventually produce Ly $\alpha$ emission. In both cases $I(\mathrm{H} \alpha) / I(\mathrm{H} \beta)=2.8$. The $I(\mathrm{Ly} \alpha) / I(\mathrm{H} \beta)$ ratio is most affected by density and takes the values 23.3 and 34.3 in the low- and high-density limits.

Spectra of low-density nebulae ionized by a hard nonthermal continuum are different from case B because of possible contributions from collisional excitation from the ground state (see Parker 1964; Netzer 1982; Ferland and Mushotzky 1982; Halpern and Steiner 1983; Gaskell and Ferland 1984). The H I spectrum produced in the ionized portion of the nebula is very close to case $\mathrm{B}$, but a strong collisional contribution occurs near the $\mathrm{H}^{+}-\mathrm{H}^{0}$ ionization front. Ionization here is predominantly by hard X-rays and the resulting suprathermal secondary electrons. As a result, the gas is heated to $10^{4} \mathrm{~K}$ in regions where hydrogen has a significant neutral fraction. A consequence is that the intensities of $\mathrm{H} \alpha$ and especially $\mathrm{Ly} \alpha$ are significantly enhanced, although the intensity of $\mathrm{H} \beta$ remains near its pure recombination value (because $1-4$ collisions are much less frequent than 1-3 or 1-2 collisions).

Gaskell and Ferland (1984) examined a large number of models of narrow-line region (NLR) gas and noted that the predictions tend to lie along a narrow band in the $\mathrm{H} \alpha / \mathrm{H} \beta$ and $\mathrm{Ly} \alpha / \mathrm{H} \beta$ plane. Here we show the physical reason why only certain values of these ratios are allowed. For a low-density gas, it is possible to separate contributions to the total line intensities into recombination and collisional components (Chamberlain 1953). It is not possible to make this separation in the high-density broad-line region because of line transfer and collisional effects involving excited states (see Netzer 1975; Osterbrock, Koski, and Phillips 1976; Kwan and Krolik 1981; Hubbard and Puetter 1984). If we write the intensity of $\mathrm{H} \alpha$ and $\mathrm{Ly} \alpha$ as the sum of recombination and collisional terms,

$$
\begin{aligned}
I(\mathrm{H} \beta) & =I(\mathrm{H} \beta)_{\mathrm{rec}}, \\
I(\mathrm{H} \alpha) & =I(\mathrm{H} \alpha)_{\mathrm{rec}}+I(\mathrm{H} \alpha)_{\mathrm{col}}, \\
I(\mathrm{Ly} \alpha) & =I(\mathrm{Ly} \alpha)_{\mathrm{rec}}+I(\mathrm{Ly} \alpha)_{\mathrm{col}},
\end{aligned}
$$

then the system of equations can be reduced to a single equation relating the intensity ratios $\mathrm{H} \alpha / \mathrm{H} \beta$ and $\mathrm{Ly} \alpha / \mathrm{H} \beta$ through a parameter proportional to the importance of collisional excitation. This is because at a given temperature the collisional contributions to $\mathrm{H} \alpha$ and $\operatorname{Ly} \alpha$ are related to each other by a simple ratio of cross sections. Aggarwal (1983) has presented rate coefficients for excitation to the first two excited states of $\mathrm{H} \mathrm{I}$, corresponding to

$$
C(1-2) / C(1-3)=2.21 t^{1 / 2} e^{2.191 / t}
$$

for the high density limit and temperatures near $t=T_{e} / 10^{4}$ $\mathrm{K} \approx 1$.

It is possible to calibrate the $\mathrm{H}$ I spectrum as an approximate reddening indicator because the temperature in regions contributing to the collisional formation of $\mathrm{H}$ lines are sharply constrained to be near $t=1$. This is basically because in cooler regions Ly $\alpha$ cannot be collisionally excited (and the spectrum will be simply case B), while much higher temperatures are not possible because cooling by collisional excitation of $\operatorname{Ly} \alpha$ would 
be larger than the heat input from photoionization. If collisional excitation of $\mathrm{Ly} \alpha$ is to be comparable to recombination, then

$$
N_{e} C_{12} N^{0} \gtrsim N_{e} N^{+} \alpha_{B}(t)
$$

where the usual notation is employed (cf. Osterbrock 1974), and we approximate $\alpha_{B}(t)$ as a power law in $t\left(\alpha_{B} \propto t^{-0.8}\right)$. Solution with standard rates (for $N_{e}>10^{4} \mathrm{~cm}^{-3}$ ) gives the lower limit

$$
t \gtrsim 11.84 /\left[11.75-\ln \left(N^{+} / N^{0}\right)+0.6 \ln (t)\right] .
$$

An upper limit to $t$ is set by the fact that cooling by collisional excitation of $\mathrm{Ly} \alpha$ cannot be greater than photoelectric heating (the actual upper limit is somewhat smaller since Ly $\alpha$ is seldom the dominant coolant in a low-density nebula). If we balance photoionization with recombination and assume that each photoionization destroys a photon with an average energy $\langle h v\rangle=5$ ryd (valid for an X-ray continuum in regions near the hydrogen ionization front) then

$$
\left(\langle h v\rangle-h v_{0}\right) N_{e} N^{+} \alpha_{B}(1) t^{-0.8}>h v_{\mathrm{Ly} \alpha} C_{12} N_{e} N^{0}
$$

with the solution

$$
t \lesssim 11.84 /\left[11.47-\ln \left(N^{+} / N^{0}\right)+0.6 \ln (t)-\ln \left(\langle h v\rangle-h v_{0}\right)\right] \text {. }
$$

Note that the only model-dependent factors are the ionization ratio $N^{+} / N^{0}$, and the mean photon energy, both of which enter as logarithms.

Model calculations for a wide range of conditions (Baldwin and Netzer 1978; Kwan and Krolik 1981; Ferland 1981; Gaskell and Ferland 1984; and unpublished), show that $N^{+} / N^{0} \approx 1-0.1$ in regions where the main collisional contribution to $\mathrm{H}$ I line formation occurs. The allowed range in temperatures, set by equations (4) and (6) assuming $\log \left(N^{+} / N^{0}\right)=0.5 \pm 1.0$, is $0.8 \lesssim t \lesssim 1.3$, where the upper limit is certainly too high because we have neglected heavy element cooling. In the following we assume a temperature $t=1$ and include the stated range of $0.8 \leq t \leq 1.2$ in our error estimate.

With $t=1$ and the rates given above, we can relate the Balmer and Lyman decrements by

$$
I(\mathrm{Ly} \alpha) / I(\mathrm{H} \beta)=a+b[I(\mathrm{H} \alpha) / I(\mathrm{H} \beta)-2.83],
$$

where the factor in parentheses at right is the enhancement of $\mathrm{H} \alpha$ above its case $\mathrm{B}$ value. The factors $a$ and $b$ are sensitive to the importance of two-photon emission as a deexcitation mechanism for $2 s$. In the high-density limit $a=34.3$ and $b=107$, while in the low-density limit $a=23.3$ and $b=67.8$.

Figure 2 shows lines of predicted Lyman and Balmer decrements (the dashed lines) for the high-and low-density cases and $t=1$. The densities inferred from such indicators as the red [S II] lines $\left(N_{e}<10^{3} \mathrm{~cm}^{-3}\right)$ and the [O III] nebular to auroral line ratio $\left(N_{e} \approx 10^{6} \mathrm{~cm}^{-3}\right.$ if $t=1$ ) (cf. Cohen and Osterbrock 1981), suggest that inhomogeneities play a fundamental role in producing NLR spectra in some objects so the density dependence presents a basic uncertainty, in addition to the model dependent electron temperature. The bold lines on the figure show the range in line ratios due to both of these effects. Predictions of many detailed models (Netzer 1982; Ferland and Mushotzky 1982; Ferland and Netzer 1983; Pequignot 1984; Netzer 1982; Halpern and Steiner 1983) lie within the permitted range in Figure 2.

Figure 2 also shows the observed line ratios in 3C 192 and

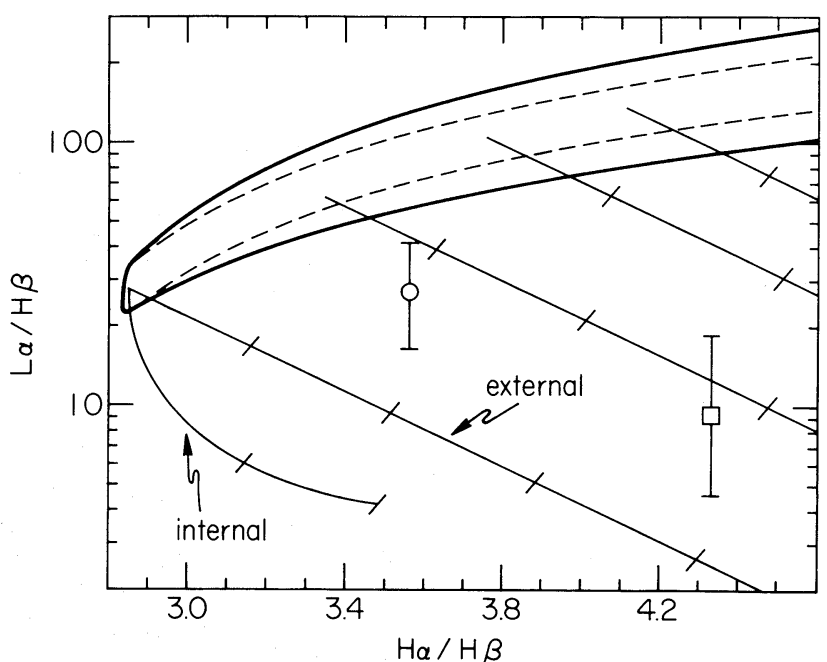

Fig. 2.-Hydrogen line intensities. The dashed lines show curves of permitted $\mathrm{H} \alpha / \mathrm{H} \beta$ and $\mathrm{Ly} \alpha / \mathrm{H} \beta$ ratios in the high- (upper) and low- (lower) density limits at a temperature of $10^{4} \mathrm{~K}$. The bold lines above and below these define the permitted range in hydrogen spectra, determined by allowing the temperature to range between 8000 and $12,000 \mathrm{~K}$. Reddening tracks, for both internal and external absorbers, are shown and are marked with large hash marks at $0.1 \mathrm{mag}$ increments in $E(B-V)$. The positions of 3C 192 (circle) and $3 C 223$ (square) are shown, along with the uncertainty in the IUE Ly $\alpha$ flux.

3C 223 along with reddening tracks away from the center of the allowed range. These curves, showing extinction due to dust along the line of sight, as well as extinction due to dust mixed with the emitting gas (taken from Ferland and Netzer 1979) are shown. It is worth stressing that reddening is the only physical process which can cause low-density gas to have both a large $\mathrm{H} \alpha / \mathrm{H} \beta$ ratio and a small $\mathrm{Ly} \alpha / \mathrm{H} \beta$ ratio (i.e., to lie in the lower right part of our diagram). Table 2 lists the reddening deduced by this method, along with the reddening corresponding to the line of sight to the NLRG through our Galaxy (Burstein and Heiles 1982). The residual reddening, which is likely to occur within the radio galaxies, is listed in the table, along with our inferred H I ratios. Apparently 3C 192 is nearly unreddened, while 3C 223 has a larger value. The intrinsic $\mathrm{H}_{\mathrm{I}}$ ratios (after correction for reddening) are quite similar and are fairly close to those predicted by models of NLR gas (Pequignot 1984).

\section{DISCUSSION}

We have argued that the intrinsic $\mathrm{H}$ I spectra of the narrowline radio galaxies 3C 192 and 3C 223 are probably similar to those predicted for X-ray photoionized nebulae. A method to

TABLE 2

INFERRED QUANTITIES

\begin{tabular}{lccc}
\hline \hline \multicolumn{1}{c}{ Quantity } & $3 \mathrm{C} 192$ & $3 \mathrm{C} 223$ & Model $^{\mathrm{a}}$ \\
\hline$E_{B-V}^{\text {tot }} \ldots \ldots \ldots$ & $0.11 \pm 0.06$ & $0.29 \pm 0.09$ & $\ldots$ \\
$E_{B-V}^{\mathrm{MW}}{ }^{\mathrm{M}} \ldots \ldots \ldots$ & 0.04 & 0.04 & $\ldots$ \\
$E_{B-V}^{\text {int }} \ldots \ldots \ldots$ & $0.07 \pm 0.06$ & $0.25 \pm 0.09$ & $\ldots$ \\
$\mathrm{Ly} \alpha / \mathrm{H} \beta^{\mathrm{d}} \ldots \ldots \ldots \ldots \ldots \ldots$. & $50 \pm 0.25 \mathrm{dex}$ & 56.9 \\
$\mathrm{H} \alpha / \mathrm{H} \beta^{\mathrm{d}} \ldots \ldots \ldots \ldots \ldots \ldots$ & $3.2 \pm 0.2$ & 3.04 \\
\hline
\end{tabular}

${ }^{\text {a }}$ Model C3b from Pequignot 1984

${ }^{b}$ Contribution from Milky Way.

' Residual reddening, presumed due to gas in the NLRG.

${ }^{d}$ Inferred intrinsic hydrogen spectrum, after correction for reddening. 
determine the interstellar reddening from measurements of $\mathrm{H} \alpha / \mathrm{H} \beta$ and $\mathrm{Ly} \alpha / \mathrm{H} \beta$, proposed by Gaskell and Ferland (1984), is developed and applied to these objects. The reddening to $3 \mathrm{C}$ 192 is only slightly larger than that due to our own Galaxy, while the extinction to $3 \mathrm{C} 223$ may be somewhat larger.

The fact that the $\mathrm{H}$ I spectra of these NLRGs are similar to those predicted for X-ray photoionized nebulae but are largely inconsistent with case B predictions suggests that the continuum ionizing the NLR gas in these objects is fairly hard. In general, the amount of collisional enhancement to $\mathrm{Ly} \alpha$ and $\mathrm{H} \beta$ is proportional to both the X-ray continuum and the metal- licity of the gas (Netzer 1982; Halpern and Steiner 1982; Halpern 1982; Gaskell and Ferland 1984). Either a hard continuum or low abundances of heavy elements tend to make neutral regions fairly hot, resulting in significant collisional enhancement.

We are grateful to the National Science Foundation for partial support of work at Lick Observatory under grant AST 83-11585, and to both the NSF and NASA for their support at the University of Kentucky (AST 83-05094 and NAG 5-239).
Aggarwal, K. M. 1983, M.N.R.A.S. 202,15P

Baldwin, J., and Netzer, H. 1978, Ap. J., 226, 1.

Burstein, D., and Heiles, C. 1982, A.J., 87, 1165.

Chamberlain, J. W. 1953, Ap. J., 117, 387 .

Cohen, R. D., and Osterbrock, D. E. 1981, Ap. J., 243, 81.

Costero, R., and Osterbrock, D. E. 1977, Ap. J., 211, 675.

Ferland, G. J. 1981, Ap. J., 249, 17.

Ferland, G. J., and Mushotzky, R. 1982, Ap. J., 262, 564.

Ferland, G. J., and Netzer, H. 1979, Ap. J., 229, 274.

- 1983, Ap. J., 264, 105.

Gaskell, C. M., and Ferland, G. J. 1984, Pub. A.S.P., 84, 393.

Halpern, J. 1982, Ph.D. thesis, Harvard University.

Halpern, J. P., and Steiner, J. E. 1983, Ap. J. (Letters), 269, L37.

\section{REFERENCES}

Hubbard, E. N., and Puetter, R. C. 1984, Ap. J., in press.

Kwan, J., and Krolik, J. 1981, Ap. J., 250, 478.

Lauer, T. R., Miller, J. S., Osborne, C. S., Robinson, L. B., and Stover, R. C. 1983, in Instrumentation in Astronomy V, Proc. Soc. Photo-Opt. Instr. Eng., $445,132$.

Netzer, H. 1975, M.N.R.A.S. 171, 395.

. 1982, M.N.R.A.S., 198, 589 .

Osterbrock, D. E. 1974, Astrophysics of Gaseous Nebulae (San Francisco: Freeman).

- 1981, Ap. J., 249, 462

Osterbrock, D. E., Koski, A. T., and Phillips, M. M. 1976, Ap. J., 206, 898

Parker, R. A. R. 1964, Ap. J., 139, 208.

Pequignot, D. 1984, Astr. Ap., 131, 159 .

Gary J. Ferland: Department of Physics and Astronomy, University of Kentucky, Lexington, KY 40506

DonAld E. Osterbrock : Lick Observatory, University of California, Santa Cruz, CA 95064 